\section{Image quality improvement in a hard X-ray projection microscope using total reflection mirror optics}

Hidekazu Mimura, ${ }^{\text {a* }}$ Kazuto Yamauchi, ${ }^{\text {a }}$ Kazuya
Yamamura, ${ }^{\text {b }}$ Akihisa Kubota, ${ }^{a}$ Satoshi Matsuyama,
Yasuhisa Sano, ${ }^{\text {a }}$ Kazumasa Ueno, ${ }^{\text {a Katsuyoshi }}$
Endo, ${ }^{b}$ Yoshinori Nishino, ${ }^{d}$ Kenji Tamasaku, ${ }^{\text {d }}$ Makina
Yabashi, ${ }^{\text {c }}$ Tetsuya Ishikawa ${ }^{d}$ and Yuzo Mori ${ }^{b}$

${ }^{a}$ Department of Precision Science and Technology, Osaka University, Yamada-Oka 2-1, Suita, Osaka 565-0871, Japan,

${ }^{b}$ Research Center of Ultraprecision Science and Technology, Osaka University, Yamada-Oka 2-1, Suita, Osaka 565-0871, Japan, 'SPring-8/Japan Synchrotron Radiation Research Institute (JASRI), Kouto 1-1-1, Mikazuki, Hyogo 679-5198, Japan, and ${ }^{d}$ SPring-8/RIKEN, Kouto 1-1-1, Mikazuki, Hyogo 679-5198, Japan.E-mail:mimura@prec.eng.osaka-u.ac.jp

A new figure correction method has been applied in order to fabricate an elliptical mirror to realize a one-dimensionally diverging $\mathrm{X}$-ray beam having high image quality. Mutual relations between figure errors and intensity uniformities of diverging X-ray beams have also been investigated using a wave-optical simulator and indicate that figure errors in relatively short spatial wavelength ranges lead to high-contrast interference fringes. By using a microstitching interferometer and elastic emission machining, figure correction of an elliptical mirror with a lateral resolution close to $0.1 \mathrm{~mm}$ was carried out. A one-dimensional diverging X-ray obtained using the fabricated mirror was observed at SPring- 8 and evaluated to have a sufficiently flat intensity distribution.

Keywords: X-ray projection microscopes; coherent X-rays; X-ray focusing; wave-optical simulation; EEM; MSI.

\section{Introduction}

The exceptional properties of X-rays have led to their use in a large variety of applications in science and technology. Recently, by utilizing X-rays obtained at third-generation synchrotron radiation facilities, the performances of such applications have advanced significantly. X-ray imaging techniques, such as holography, phasecontrast imaging and refraction-contrast imaging are powerful methods for the non-destructive analysis of inner structures of materials in various fields including medical, biological and materials science (Illinski et al., 2003; Bohic et al., 2001; Wilhein et al., 2001). In $\mathrm{X}$-ray imaging applications with high spatial resolution, a projection geometry set-up is often employed. Focusing optics, such as Fresnel zone plates (Suzuki et al., 2001) and focusing mirrors (Hignette et al., 2001; Ice et al., 2000; Mori et al., 2002), are utilized to illuminate samples by diverging X-ray beams.

Total reflection mirrors are attractive optics from the viewpoints of having no chromatic aberration and high-intensity beams. By employing focusing mirror optics to obtain diverging X-ray beams, the wavelength of the X-rays can be changed in the same geometric arrangement. However, when using mirrors fabricated by conventional methods, high-contrast interference fringes invariably appear in diverging X-ray beam images. The appearance of such fringes is the reason why focusing mirrors have not been utilized in projection microscopes.
We have established a new fabrication system of X-ray mirrors constructed by plasma chemical vaporization machining (PCVM) (Mori, Yamamura \& Sano, 2000; Mori, Yamauchi et al., 2000), elastic emission machining (EEM) (Yamauchi, Mimura et al., 2002; Mori et al. 1987, 1988) and microstitching interferometry (MSI) (Yamauchi et al., 2003). This system can yield mirror surfaces with peak-to-valley (PV) accuracies as high as $1 \mathrm{~nm}$ and a lateral resolution close to $0.1 \mathrm{~mm}$. In the previous study, a flat mirror, having a PV accuracy higher than $1 \mathrm{~nm}$ over a spatial wavelength range longer than $0.5 \mathrm{~mm}$, was fabricated and evaluated to have a sufficiently flat intensity distribution in a totally reflected beam of wavelength $0.06 \mathrm{~nm}$ at the $1 \mathrm{~km}$-long beamline BL29XUL of SPring-8 (Mori, Yamauchi, Yamamura, Mimura et al., 2002; Ishikawa et al., 2001). In addition, a focusing mirror having an elliptical shape was also fabricated and certified to have a diffraction-limited focusing performance at the same beamline (Yamamura et al., 2003; Yamauchi, Yamamura, Mimura et al., 2002, 2003). The focal beam profile was confirmed to agree well with beam profiles predicted by calculating the FresnelKirchhoff integral, not only in focal size but also in the shape of the intensity distribution, including satellite peak structures. These results support the effectiveness of the proposed system.

In this study the fabrication technologies were applied to construct a highly accurate elliptical mirror in order to realize a one-dimensional diverging X-ray from the focal point as a line source. The mutual relations between intensity uniformities of diverging X-ray beams and the nature of the figure errors of elliptical mirrors were investigated by utilizing a wave-optical simulator. The obtained results show that figure errors in relatively short spatial wavelength ranges, such as shorter than $1 \mathrm{~mm}$, lead to high-contrast interference fringes. In order to remove such fringes, figure correction of lateral resolution $0.3 \mathrm{~mm}$ was performed on focusing mirror surfaces by EEM and MSI. One-dimensional diverging X-ray beams were observed at BL29XUL and evaluated to reduce the fluctuation to $10 \%$ of the averaged intensity.

\section{Relation between the uniformity of intensity distributions in diverging $\mathrm{X}$-ray beams and the figure error profiles on elliptical mirrors}

The cause of the interference fringes in the intensity distribution image of diverging X-ray beams obtained by focusing mirrors are the figure errors of the employed mirror surfaces. In order to understand the mutual relation between the intensity uniformities and the nature of the figure errors, wave-optical simulations, which can estimate the intensity distribution profiles of totally reflected beams by calculating the Fresnel-Kirchhoff integrals while taking mirror surface profiles into account (Mori, Yamauchi, Yamamura, Mimura et al., 2002; Born \& Wolf, 1997), were carried out. The geometrical relations between the X-ray source, mirror and screen in the simulator were set up as shown schematically in Fig. 1. This configuration was the same as that of BL29XUL. The shape of the investigated mirror was an ellipse, having a length of $100 \mathrm{~mm}$ and a focal distance of $300 \mathrm{~mm}$. The figure profile and ellipse parameters are shown in Fig. 2 and Table 1. The incident X-ray energy and average glancing angle were set to $15 \mathrm{keV}$ and $1.4 \mathrm{mrad}$, respectively. The incident slit, of width $100 \mu \mathrm{m}$, was located $1 \mathrm{~km}$ upstream of the mirror position. Fig. 3 shows the calculated intensity distribution profile of diverging X-ray beams at $600 \mathrm{~mm}$ downstream of the focal point, when the figure of the mirror is ideal.

Three ellipse profiles having figure errors characterized in short, middle and long spatial wavelength ranges were prepared and input into the simulator as mirror surface profiles. These error profiles, 


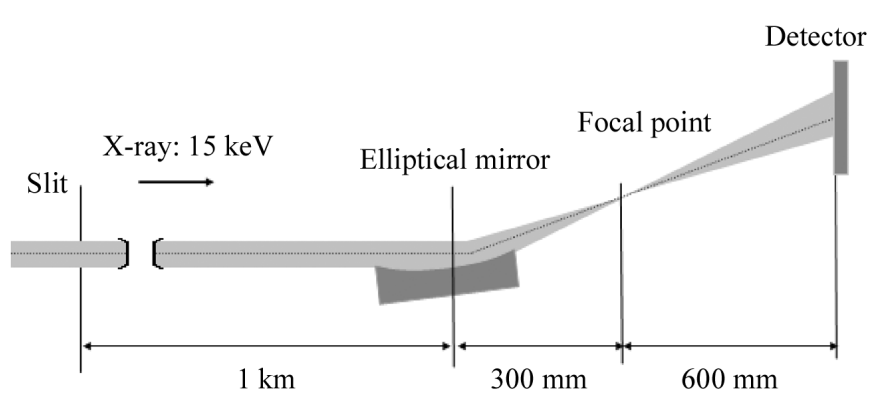

Figure 1

Schematic drawing of the geometrical relations between the X-ray source, mirror and screen in the simulator.

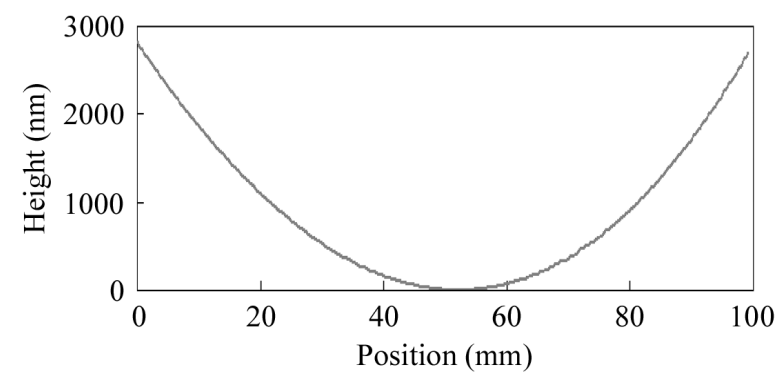

Figure 2

Surface profile of the elliptical mirror.

having RMS figure errors of $0.1 \mathrm{~nm}$, are shown in Figs. 4(a)-4(c). The short, middle and long spatial wavelength ranges correspond to $0.1-$ $2 \mathrm{~mm}, 2-50 \mathrm{~mm}$ and $50-100 \mathrm{~mm}$, respectively. Figs. 5(a)-5(c) show three calculated intensity distribution profiles. Sharp fluctuations appear in the profile of the beam reflected by the mirror having a figure error in the short spatial wavelength range. In the case of the figure error in the middle spatial wavelength range, such fluctuations disappeared in the intensity profile. The intensity profile concerning the long spatial wavelength range is almost the same as the ideal one. From a comparison with previous reports (Yamauchi, Yamamura, Mimura et al., 2002) investigating the mutual relation between the uniformities of a focused beam profile and the nature of figure errors, it may be concluded that the required figure accuracy to realize ideal diverging $\mathrm{X}$-ray beams is higher than that required to realize diffraction-limited focusing. In particular, unprecedented degrees of figure accuracy are required in the short spatial wavelength ranges.

\section{Fabrication of an elliptical mirror to realize the ideal diverging $\mathrm{X}$-ray beams}

In order to remove the high-contrast interference fringes from the diverging X-ray beam, figure correction having a lateral resolution close to $0.1 \mathrm{~mm}$ was carried out on an elliptical mirror surface by EEM and MSI. EEM is an ultraprecise machining method that utilizes the chemical reaction between the surfaces of the workpiece and those of the fine powder particles that react with the workpiece material. The fine powder particles are mixed with ultrapure water and transported to the workpiece surface in a controlled flow. When the surfaces of the workpiece and the powder particles come into contact with each other and are subsequently separated, there is a notable probability that the topmost atoms on the workpiece will preferentially adhere to and thus move onto the surface of the power particle. In numerically controlled (NC) machining, nozzle-type EEM heads generate high shear-rate flows of ultrapure water on the workpiece surface in order to transport fine powder particles. Using
Table 1

Parameters of the focusing mirror having a $300 \mathrm{~mm}$ focal length.

\begin{tabular}{ll}
\hline Substrate material & CZ-(111)Si single crystal \\
Surface coating & None \\
Effective mirror size in longitudinal direction & $90 \mathrm{~mm}$ \\
Length of ellipse & $500.15 \mathrm{~m} \times 2$ \\
Breadth of ellipse & $24.25 \mathrm{~mm} \times 2$ \\
Focus length & $300 \mathrm{~mm}$ \\
Glancing angle on optical axis & $1.40 \mathrm{mrad}$ \\
Maximum glancing angle & $1.55 \mathrm{mrad}$ \\
Acceptance width & $130 \mu \mathrm{m}$ \\
\hline
\end{tabular}

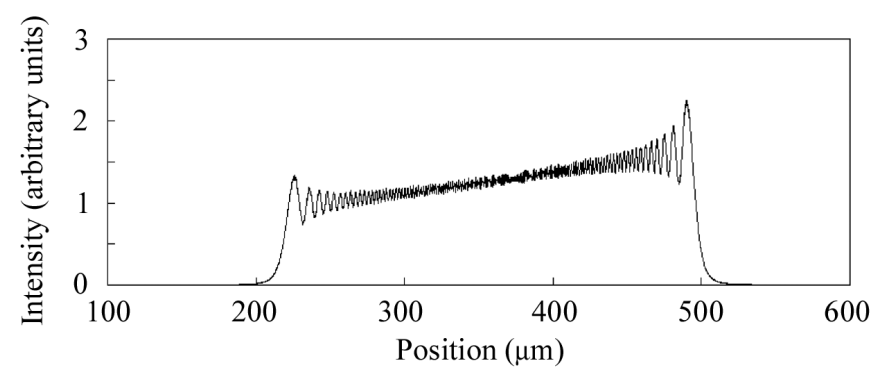

Figure 3

Calculated intensity profile of diverging X-ray beams $600 \mathrm{~mm}$ downstream of the focal point, using an ideal ellipse profile.

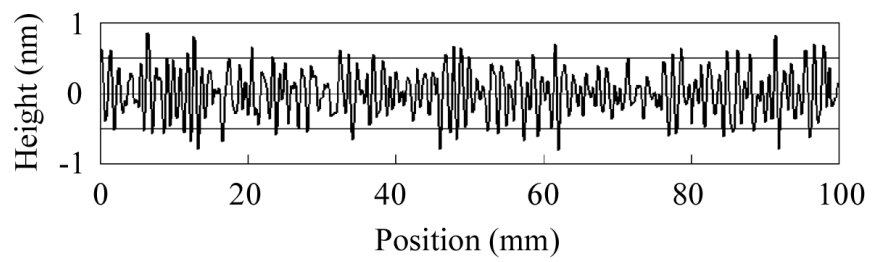

(a)

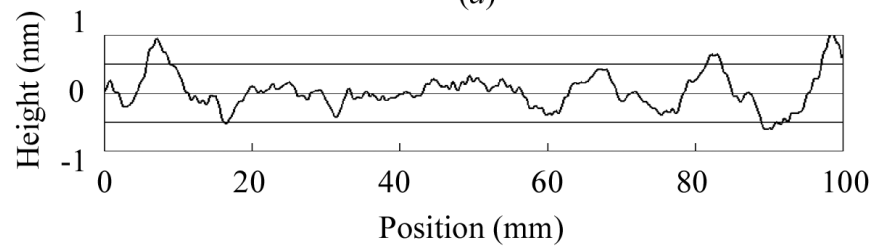

(b)

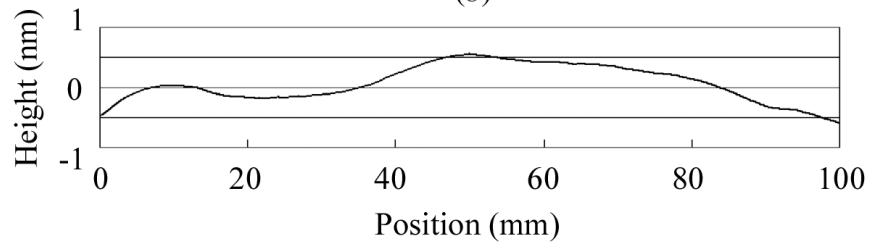

(c)

Figure 4

Figure error profiles characterized in $(a)$ short, $(b)$ middle and $(c)$ long spatial wavelength ranges to be used in the investigation of the relation between the nature of figure errors and the uniformity of diverging X-ray beams.

this type of EEM head, the lateral resolution in figure correction can be adjusted by selecting a suitable nozzle aperture according to the required lateral resolution. The finest lateral resolution typically realized in NC EEM is $0.3 \mathrm{~mm}$ when a nozzle aperture of diameter $0.15 \mathrm{~mm}$ is employed (Yamauchi, Mimura et al., 2002).

MSI, in which a microscopic and a large-area phase-shifting interferometer are compositely utilized, is employed for figure profiling. This method can measure surface profiles with a PV accu- 
racy higher than $1 \mathrm{~nm}$ and with a spatial resolution close to $10 \mu \mathrm{m}$ (Yamauchi et al., 2003).

The preprocessed surface was prepared by NC PCVM. Fig. 6(a) shows the residual figure error profile of the preprocessed surface. The objective profile is the same as the profile shown in Fig. 2. The PV

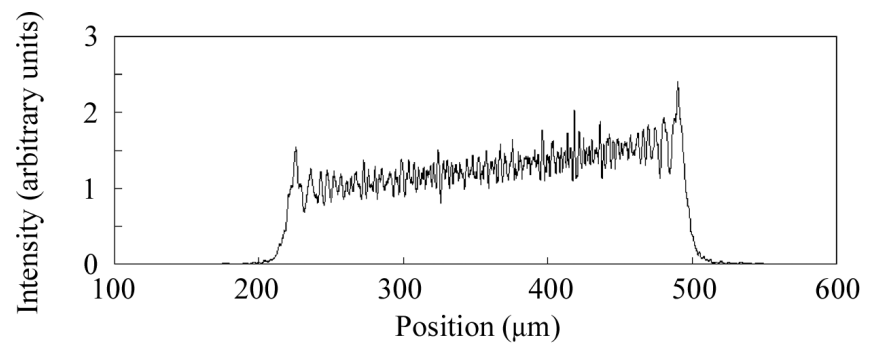

(a)

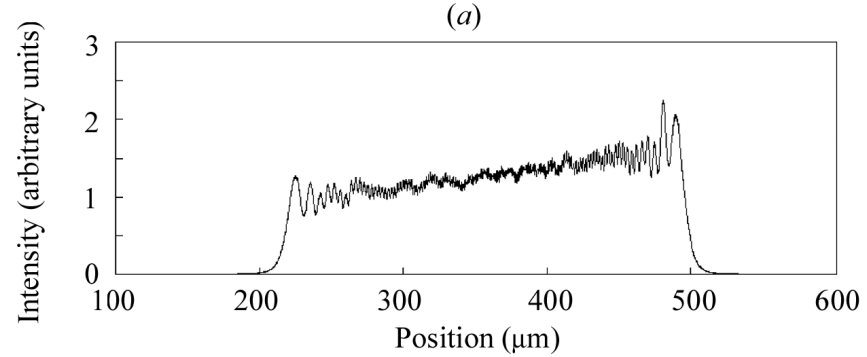

(b)

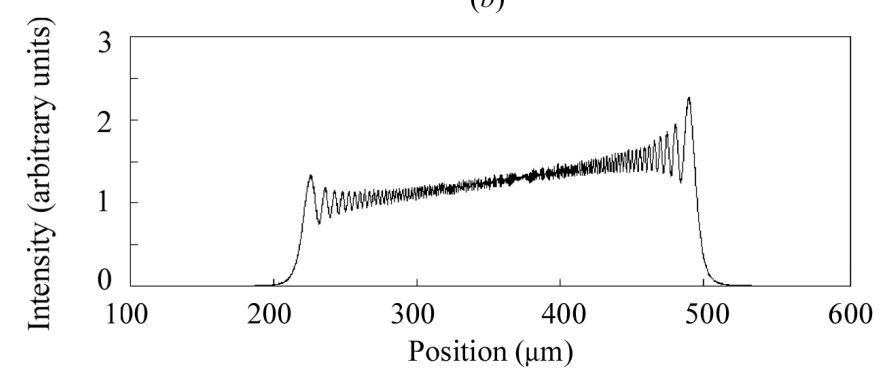

(c)

\section{Figure 5}

Calculated intensity profiles of diverging X-ray beams using the elliptical mirror having figure errors characterized in $(a)$ short, $(b)$ middle and $(c)$ long spatial wavelength ranges.

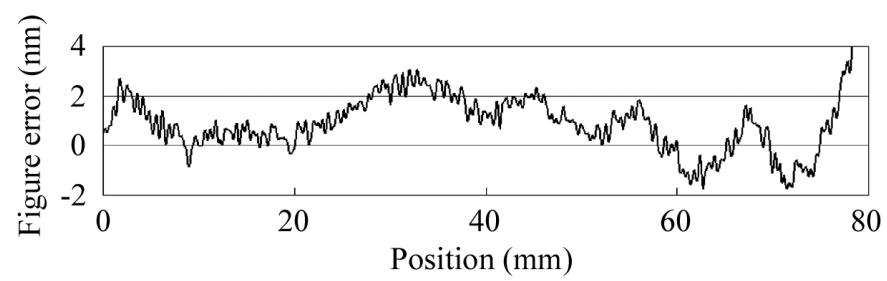

(a)

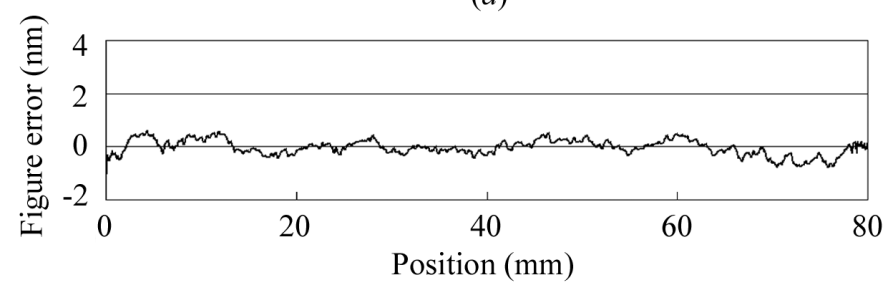

(b)

Figure 6

Residual figure error profiles of $(a)$ preprocessed and (b) EEM-processed surfaces. and RMS figure errors of the preprocessed surface are $6.5 \mathrm{~nm}$ and $1.2 \mathrm{~nm}$, respectively. Fig. 6(b) shows the residual figure error profile of the EEM-processed surface. After the EEM process, the PV and RMS figure errors obtained were $2.6 \mathrm{~nm}$ and $0.2 \mathrm{~nm}$, respectively. In particular, it is found that the figure errors characterized in the relatively short spatial wavelength ranges were removed. Fig. 7 shows the result of power spectral density analysis of preprocessed and EEM-processed surface profiles and indicates that figure correction was carried out in spatial wavelength ranges longer than $0.3 \mathrm{~mm}$.

\section{Evaluation of diverging X-ray images}

By focusing on the flatness of the image of a one-dimensional diverging X-ray beam, the fabricated mirror was evaluated at BL29XUL. In the investigation, another mirror having different surface qualities was prepared and compared with the EEM-finished mirror to confirm the simulation results. The residual figure error profile of the mirror is shown in Fig. 8. This mirror was prepared by NC EEM having a lateral resolution of $2 \mathrm{~mm}$.

The optical system in the experimental set-up for observing diverging X-ray beams is the same as that shown in Fig. 1. Monochromatic X-rays at $15 \mathrm{keV}$ were prepared using a cryogenic-cooled double-crystal Si 111 monochromator (Mochizuki et al., 2001; Kitamura, 1998) and guided to the experimental hutch of the beamline located $1 \mathrm{~km}$ downstream of the centre of the light-source undulator (Hrdý, 1998). An X-ray zooming tube and CCD camera system (HAMAMATSU, C533) were employed to observe the beam images. The focused beam sizes of the two mirrors were previously confirmed to be $0.18 \mu \mathrm{m}$ in FWHM and to be in diffraction-limited conditions.

Figs. $9(a)$ and $9(b)$ show the intensity distribution images of the diverging X-ray beams reflected by the EEM-finished and addition-

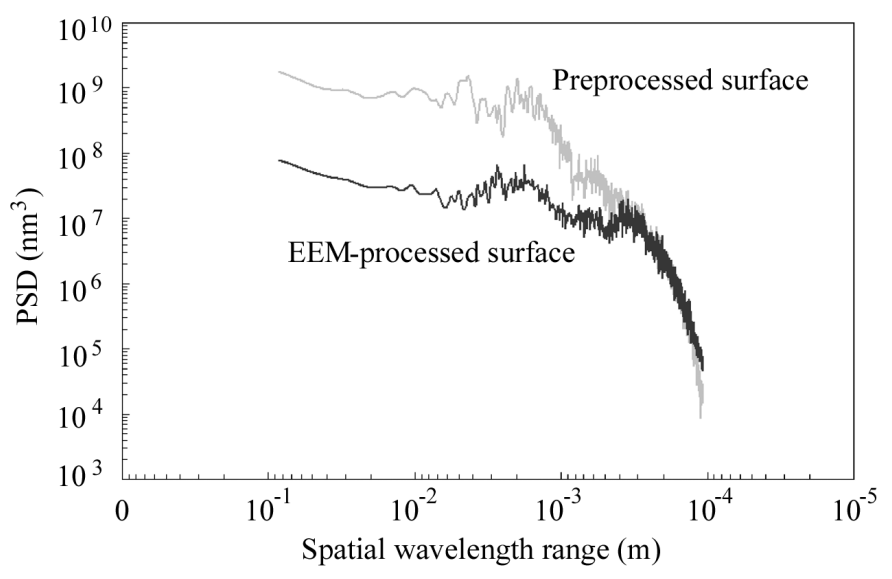

Figure 7

Power spectral density curves of residual figure errors of preprocessed and EEM-processed surfaces.

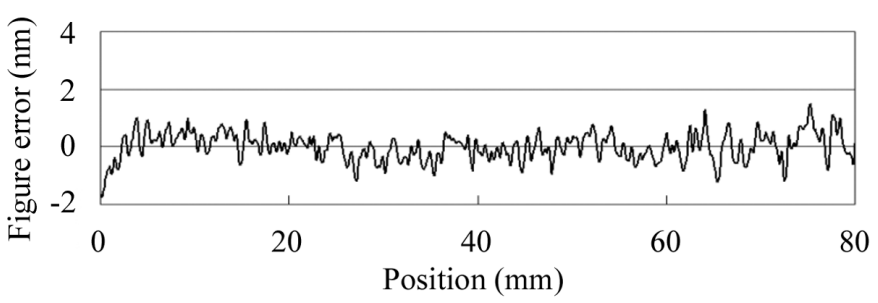

Figure 8

Residual figure error profile of the mirror, the figure error of which is not corrected in short spatial wavelength ranges. 


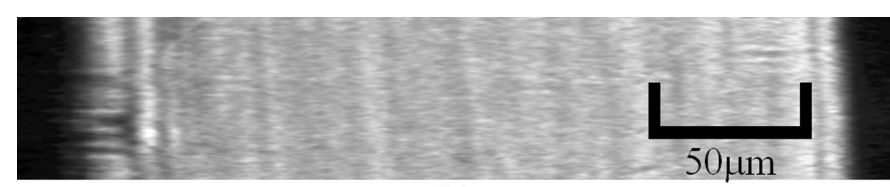

(a)

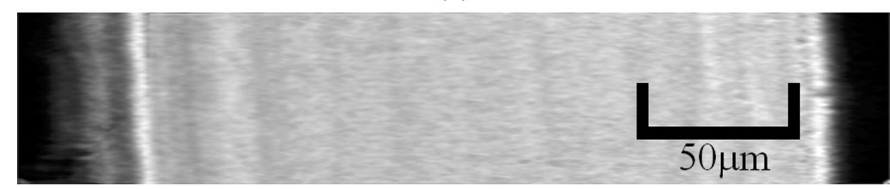

(b)

\section{Figure 9}

Intensity distribution images of the diverging X-ray beams reflected by the EEM-finished mirror and by another mirror having the surface figure error profiles shown in Fig. 8. (a) Mirror having the surface figure error profiles shown in Fig. 8. (b) EEM-finished mirror.

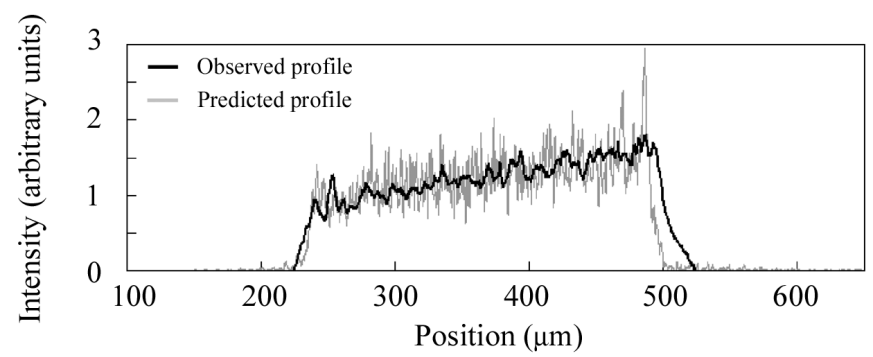

(a)

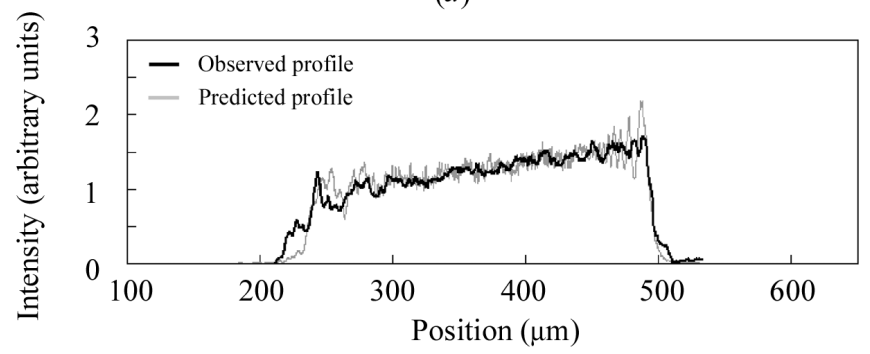

(b)

\section{Figure 10}

Observed and predicted intensity distribution profiles of the diverging X-ray beams reflected by the mirror having the surface figure error profiles shown in Fig. 8 and by the EEM-finished mirror. (a) Mirror having the surface figure error profiles shown in Fig. 8. (b) EEM-finished mirror.

ally prepared mirrors, respectively. The intensity distribution profiles in the two images are shown in Figs. 10(a) and 10(b), together with intensity profiles predicted by the wave-optical simulator in which the elliptical surface profiles having figure errors shown in Fig. $6(b)$ and Fig. 8 were used as the boundary conditions.

The observed intensity profile of the EEM-finished surface fluctuates with an amplitude of $10 \%$ of the averaged intensity, except for the edge of the profile, and agrees well with the predicted profile. In contrast, the observed profile of the mirror, of which the figure error is not corrected in the short spatial wavelength range, fluctuates with an amplitude of more than $30 \%$; however, it does not agree well with the predicted one because of the spatial resolution and sensitivity of the employed detector. Meanwhile, we can conclude that the sharp fluctuations can be removed by performing figure corrections having high spatial resolutions close to $0.1 \mathrm{~mm}$ because of the agreement between the observed and calculated profiles of the finely finished EEM surface.

This research was partially supported by the Ministry of Education, Science, Sports and Culture, Grant-in-Aid for Scientific Research (S), 151060032003 and the 21st Century COE Program 2003.

\section{References}

Bohic, S., Simionovici, A., Snigirev, A., Ortega, R., Deves, G., Heymann, D. \& Schroer, C. G. (2001). Appl. Phys. Lett. 78, 3544-3546.

Born, M. \& Wolf, E. (1997). Principles of Optics, 6th ed. Cambridge University Press.

Hignette, O., Rostaing, G., Cloetens, P., Rommeveaux, A., Ludwig, W. \& Freund, A. K. (2001). Proc. SPIE, 4499, 105-116.

Hrdý, J. (1998). J. Synchrotron Rad. 5, 1206-1210.

Ice, G. E., Chung, J. S.,, Tischler, J. Z., Lunt, A. \& Assoufid, L. (2000). Rev. Sci. Instrum. 71, 2635-2639.

Illinski, P., Lai, B., Cai, Z., Yun, W., Lengnini, D., Talarico, T., Cholewa, M., Webster, L. K., Deacon, G. B., Rainone, S., Phillips, D. E. \& Stanpfl, A. P. J. (2003). Cancer Res. 63, 1776-1779.

Ishikawa, T., Tamasaku, K., Yabashi, M., Goto, S., Tanaka, Y., Yamazaki, H., Takeshita, K., Kimura, H., Ohashi, H., Matsushita, T. \& Ohata, T. (2001). Proc. SPIE, 4145, 1-10.

Kitamura, H. (1998). J. Synchrotron Rad. 5, 184-188.

Mochizuki, T., Kohmura, Y., Awaji, A., Suzuki, Y., Baron, A., Tamasaku, K., Yabashi, M., Yamazaki, H. \& Ishikawa, T. (2001). Nucl. Instrum. Methods, A467/468, 647-649.

Mori, Y., Yamamura, K. \& Sano, Y. (2000). Rev. Sci. Instrum. 71, 4620-4626.

Mori, Y., Yamauchi, K. \& Endo, K. (1987). Precis. Eng. 9, 123-128.

Mori, Y., Yamauchi, K. \& Endo, K. (1988). Precis. Eng. 10, 24-28.

Mori, Y., Yamauchi, K., Yamamura, K., Mimura, H., Sano, Y., Saito, A., Ueno, K., Endo, K., Souvorov, A., Yabashi, M., Tamasaku, K. \& Ishikawa, T. (2002). Proc. SPIE, 4782, 58-64.

Mori, Y., Yamauchi, K., Yamamura, K. \& Sano, Y. (2000). Rev. Sci. Instrum. 71, $4627-4632$

Suzuki, Y., Takeuchi, A., Takano, H., Ohigashi, T. \& Takenaka, H. (2001). Proc. SPIE, 4499, 74-83.

Wilhein, T., Kaulich, B., Fabrizio, E., Romanato, F., Cabrini, S. \& Susini, J. (2001). Appl. Phys. Lett. 78, 2082-2084.

Yamauchi, K., Mimura, H., Inagaki, K. \& Mori, Y. (2002). Rev. Sci. Instrum. 73, $111-117$.

Yamauchi, K., Yamamura, K., Mimura, H., Sano, Y., Kanaoka, M., Endo, K., Souvorov, A., Yabashi, M., Tamasaku, K., Ishikawa, T. \& Mori, Y. (2002). Proc. SPIE, 4782, 271-276.

Yamauchi, K., Yamamura, K., Mimura, H., Sano, Y., Saito, A., Souvorov, A., Yabashi, M., Tamasaku, K., Ishikawa, T. \& Mori, Y. (2002). J. Synchrotron Rad. 9, 313-316.

Yamauchi, K., Yamamura, K., Mimura, H., Sano, Y., Saito, A., Ueno, K., Endo, K., Souvorov, A., Yabashi, M., Tamasaku, K., Ishikawa, T. \& Mori, Y. (2003). Jpn. J. Appl. Phys. 42, 7129-7134.

Yamauchi, K., Yamamura, K., Mimura, H., Sano, Y., Saito, A., Ueno, K., Endo, K., Souvorov, A., Yabashi, M., Tamasaku, K., Ishikawa, T. \& Mori, Y. (2003). Rev. Sci. Instrum. 74, 2894-2898.

Yamamura, K., Yamauchi, K., Mimura, H., Sano, Y., Saito, A., Endo, K., Souvorov, A., Yabashi, M., Tamasaku, K., Ishikawa, T. \& Mori, Y. (2003). Rev. Sci. Instrum. 74, 4549-4553. 